\title{
Editorial
}

Ocular Oncology

and Pathology
Ocul Oncol Pathol 2017;3:259-261

DOI: $10.1159 / 000461586$
Received: October 19, 2016

Accepted after revision: February 7, 2017

Published online: March 24, 2017

\section{Is There an Increased Prevalence of Asteroid Hyalosis in Eyes with Uveal Melanoma? A Histopathologic Study}

\author{
Christopher K.H. Burris ${ }^{a}$ Amir A. Azari ${ }^{a}$ Mozhgan R. Kanavi ${ }^{b}$ \\ Richard R. Dubielzig ${ }^{c}$ Vivian Lee ${ }^{a} \quad$ Justin L. Gottlieb ${ }^{a}$ Heather D. Potter ${ }^{a}$ \\ Kyungmann Kim ${ }^{d}$ Meisha L. Raven ${ }^{a}$ Maria E. Rodriguez ${ }^{a} \quad$ Devasis N. Reddy $^{a}$ \\ Daniel M. Albert ${ }^{\mathrm{e}}$ \\ ${ }^{a}$ Department of Ophthalmology and Visual Sciences, University of Wisconsin, Madison, WI, USA; ${ }^{\mathrm{b}}$ Ocular Tissue \\ Engineering Research Center, Shahid Beheshti University of Medical Sciences, Tehran, Iran; 'C Comparative Ocular \\ Pathology Laboratory of Wisconsin, University of Wisconsin School of Veterinary Medicine, and d Department of \\ Biostatistics and Informatics, University of Wisconsin, Madison, WI, and e Casey Eye Institute, Oregon Health and \\ Science University, Portland, OR, USA
}

\section{Keywords}

Asteroid hyalosis · Uveal melanoma · Prevalence

\begin{abstract}
During the planning meeting for the Collaborative Ocular Melanoma Study (COMS) prior to the start of patient recruitment in 1986, there was an interest expressed in determining whether a relationship existed between the presence of uveal melanoma (UM) and asteroid hyalosis $(\mathrm{AH})$. To answer this question, the ophthalmic examination form (unlike the pathology form for enucleated eyes) for each COMS patient asked whether $\mathrm{AH}$ was present or not. Though an increased prevalence was not found, this result was never published. A recent unpublished study at the University of Wisconsin School of Veterinary Medicine indicated a higher prevalence of $\mathrm{AH}$ in canine eyes with UM when compared to control eyes (without tumor) enucleated for goniodysgenesis. This further increased our inter-
\end{abstract}

\section{KARGER}

(C) 2017 S. Karger AG, Basel

E-Mail karger@karger.com

www.karger.com/oop est in revisiting the published literature, clinical records, and histopathology slides of the enucleated eyes from the COMS study, as well as the histopathology slides on file in the University of Wisconsin Eye Pathology Laboratory. While cases with both $\mathrm{AH}$ and UM were occasionally encountered in the literature, clinically, we could not find a previous study focusing on these two processes. This study was conducted to explore whether such an association exists.

(c) 2017 S. Karger AG, Basel

\section{Background}

Asteroid hyalosis ( $\mathrm{AH})$ is a degenerative and age-related process in the vitreous of unknown etiology with a reported clinical and histological prevalence ranging from 0.36 to $2.9 \%$ [1-3]. Histopathologically, the asteroid bodies appear as basophilic crystalline spherules that stain 


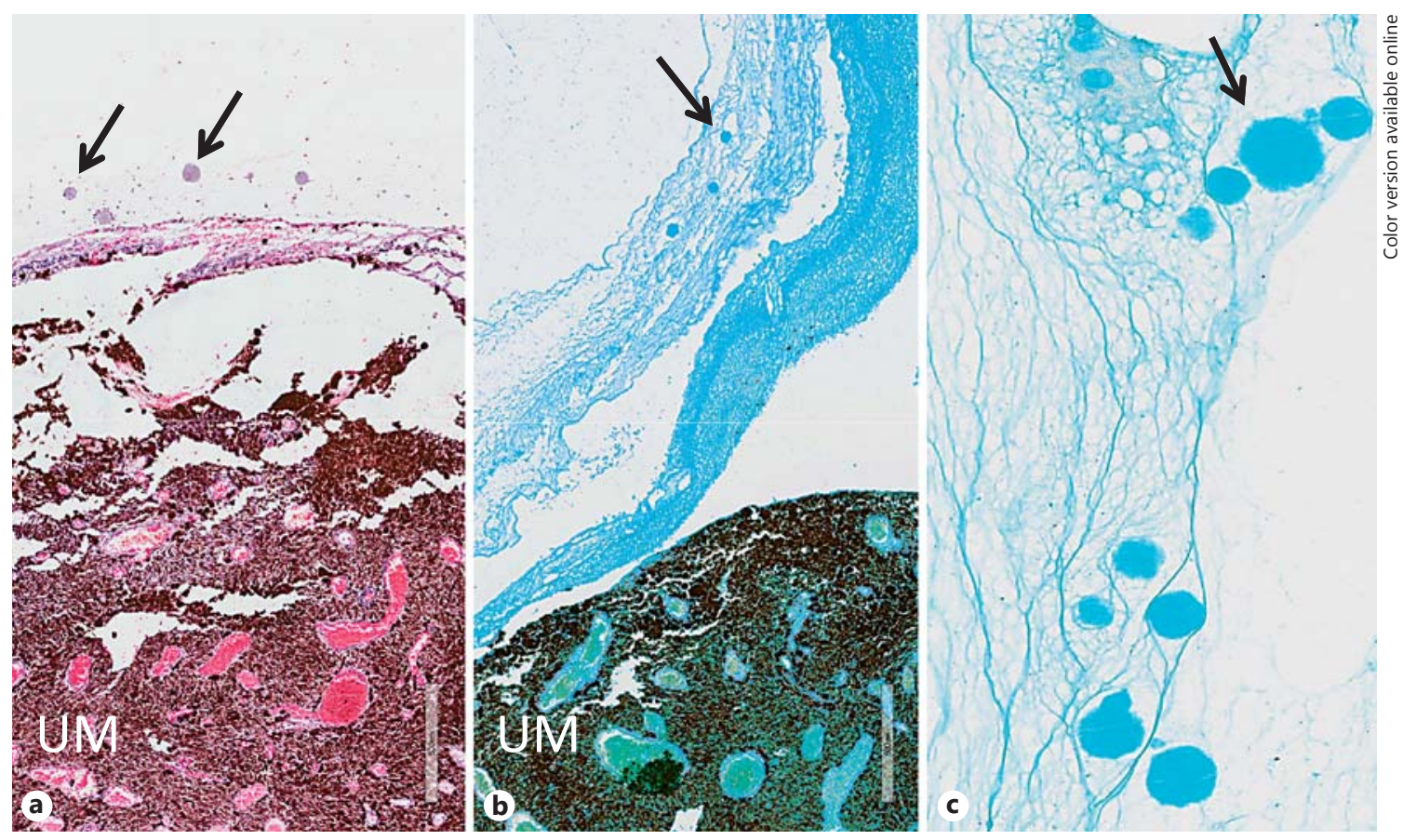

Fig. 1. Images depict round spherules characteristic of asteroid hyalosis (arrows) in an eye with uveal melanoma $(\mathrm{UM})$ on hematoxylin and eosin $(\mathbf{a}$, original magnification $\times 100)$, stained blue with Alcian blue staining $(\mathbf{b}$, original magnification $\times 100$; c, original magnification $\times 400)$.

positively for acid mucopolysaccharide, and are birefringent with a Maltese cross pattern under polarized light.

Uveal melanoma (UM) has an incidence of 7 cases per million per year in the United States, making it the most common primary intraocular malignancy in adults. The mean age at presentation is 59 years old and it affects both sexes equally [4]. Its etiology is unknown, but many studies have given insight into the associated chromosomal and genetic changes.

\section{Materials and Methods}

The clinical prevalence of $\mathrm{AH}$ in eyes with $\mathrm{UM}$ was determined by reviewing the clinical records of eyes enucleated during the Collaborative Ocular Melanoma Study (COMS). As a control, the clinical prevalence of $\mathrm{AH}$ was established from patients without UM seen in the retina department in the University of Wisconsin Eye Clinic by a single observer (J.L.G.), who systematically documented the presence of $\mathrm{AH}$ in his patients. The clinical association between UM and $\mathrm{AH}$ was determined by comparing the prevalence between the two groups and compared using a two-sample proportion test.

The histopathological prevalence in eyes with UM was obtained by reviewing archived histology slides and specimens from the COMS and eyes with UM on file in the University of Wisconsin Eye Pathology Laboratory. Multiple hematoxylin and eosin slides in each case were examined by several experienced reviewers (M.R.K., A.A.A., H.D.P., V.L., R.R.D., and D.M.A.), and cases with a sufficient amount of vitreous had the vitreous screened for the presence of basophilic crystalline spherules. The unstained slides in suspicious cases were stained with Alcian blue. The spherules were confirmed to be AH with Alcian blue staining and polarization (Fig. 1a-c).

\section{Results}

Review of the clinical records of 1,548 eyes from the COMS revealed a clinical prevalence of $1.2 \%$ (19 of 1,548 eyes) amongst eyes with UM. In the control group, the clinical prevalence of $\mathrm{AH}$ in eyes without $\mathrm{UM}$ was $0.82 \%$ (43 of 5,218 eyes). This 1.5 -fold increase in the clinical prevalence of AH in UM eyes, when compared to the controls was not found to be statistically significant (95\% CI $0.871-2.55, p=0.19)$.

There were a total of 1,985 UM histopathology specimens (1,759 eyes from the COMS and 226 eyes from the eye pathology laboratory at the University of Wisconsin); 
out of these, 28 (1.4\%) cases had anterior uveal involvement and 1,957 (98.6\%) cases had only posterior uveal involvement. The histological prevalence of $\mathrm{AH}$ amongst the COMS and University of Wisconsin eyes was $0.7 \%$ (13 of 1,985 eyes).

\section{Discussion}

$\mathrm{AH}$ is a complex but poorly understood entity associated with a number of biochemical changes in the eye. It has been suggested that it is caused by toxins diffusing into the vitreous from degenerating retina $[5,6]$. It is well demonstrated that the prevalence of both $\mathrm{AH}$ and UM increases with age $[2,7,8]$. While the prevalence of $\mathrm{AH}$ in our control group was comparable to previously reported rates, including the largest study from Bergren et al. [9], it was less than the rates found in the Beaver Dam Eye Study and a study performed at the University of California at Los Angeles (UCLA) [2, 3]. These differences can be explained by the different data collection and investigation methods employed by each study. Additionally, patients from the Beaver Dam and UCLA studies were older than our control group, which may have contributed to the higher prevalence in those studies.

As with all retrospective clinical studies, there were several limitations. We did not have a histological control group and due to the sampling of different groups, the clinical versus histological prevalence of $\mathrm{AH}$ was unable to be assessed via McNemar's test. Secondly, clinical detection of $\mathrm{AH}$ by the examination of a single clinician may not have the same sensitivity as examination by several clinicians. Part of the increased clinical prevalence of $\mathrm{AH}$ in eyes with UM versus controls may reflect more thorough posterior segment examinations and/or more detailed record keeping in eyes with known UM. Histological examination of the vitreous has its own pitfalls, since much of the vitreous is lost during processing. This may account for our clinical detection rate being higher than our histological rate in eyes with UM. Furthermore, there is a possibility that the patients in either of our groups had other systemic and or ocular conditions contributing to $\mathrm{AH}$. In conclusion, there does not appear to be a statistically significant association between AH and UM.

\section{Acknowledgements}

We thank Vicky Rogness, Kristina Lund, and Kate Lieber for their assistance with this project. This work was supported by the National Institutes of Health grant P30-EY016665 (Core Grant for Vision Research) and an unrestricted award from the Research to Prevent Blindness.

\section{Statement of Ethics}

The authors have no ethical conflicts to disclose.

\section{Disclosure Statement}

The authors have no conflicts of interest.

\section{References}

$1 \mathrm{Kim} \mathrm{JH}$, Roh MI, Byeon SH, et al: Prevalence of and risk factors for asteroid hyalosis in Seoul, Korea. Retina 2008;28:1515-1521.

2 Fawzi AA, Vo B, Kriwanek R, et al: Asteroid hyalosis in an autopsy population: The University of California at Los Angeles (UCLA) experience. Arch Ophthalmol 2005;123:486490.

3 Moss SE, Klein R, Klein BEK: Asteroid hyalosis in a population: the Beaver Dam Eye Study. Am J Ophthalmol 2001;132:70-75.
4 Eagle RC Jr: Intraocular tumors in adults. In: Tasman W: Duane's Foundations of Clinical Ophthalmology 2004; volume 3, chapter 20, Lippincott Williams and Wilkins, on CDROM.

5 Kador PF, Wyman M: Asteroid hyalosis: pathogenesis and prospects for prevention. Eye 2008;22:1278-1285.

6 Zigler JS Jr, Hess HH: Cataracts in the Royal College of Surgeons rat: evidence for initiation by lipid peroxidation products. Exp Eye Res 1985;41:67-76.
7 Bergman L, Seregard S, Nilsson B, Ringborg U, Ragnarsson-Olding B: Incidence of uveal melanoma in Sweden from 1960 to 1998. Invest Ophthalmol Vis Sci 2002;43:2579-2583.

8 Singh AD, Bergman L, Seregard S: Uveal melanoma: epidemiologic aspects. Ophthalmol Clin North Am 2005;18:75-84.

9 Bergren RL, Brown GC, Duker JS: Prevalence and association of asteroid hyalosis with systemic diseases. Am J Ophthalmol 1991;111: 289-293. 